\title{
EL SIMBOLISMO PUNITIVO EN LA PREVENCIÓN Y REPARACIÓN DE DAÑOS DERIVADOS DE LAS MEDIDAS DE PROTECCIÓN
}

\author{
PUNITIVE SYMBOLISM IN THE PREVENTION AND REPAIR OF \\ DAMAGES DERIVED FROM PROTECTIVE MEASURES
}

\author{
Manuel Bermúdez Tapia* \\ Universidad Privada San Juan Bautista \\ Lima-Perú \\ http:/ /orcid.org/0000-0003-1576-9464
} manuel.bermudez@upsjb.edu.pe mbermudeztapia@gmail.com

Nuccia Seminario Hurtado** Investigadora independiente Lima-Perú https: / / orcid.org/0000-0002-1805-7780 nuccia_sh@hotmail.com

\section{Resumen}

El contexto de violencia social incide en el ámbito familiar generando el incremento desproporcionado de las estadísticas en los últimos años haciendo estéril las acciones del Estado a través de sus entidades públicas. Las políticas públicas de control de actos de violencia en el interior de las familias están siendo desacreditada y la verdadera razón de este fracaso detalla un defecto de fondo: el Estado está asumiendo que la violencia se combate a través de acciones legales, judiciales y estatales, con lo cual se evita atender el verdadero origen de la violencia extrema en el país.

Como resultado de esta situación es que se observa una legislación que se modifica en forma constante, estableciéndose más niveles coercitivos y punitivos pero sin atender a los hechos que provocan las situaciones de violencia, agresividad y descontrol de las personas en sus relaciones familiares, porque además estas se han transformado en los últimos años, generando un sistema normativo simbólico y disfuncional que condiciona

Abogado por la Pontificia Universidad Católica del Perú. Magister en Derecho, Doctorado en Derecho por la Pontificia Universidad Católica de Argentina. Profesor Investigador de la Universidad Privada San Juan Bautista y profesor de la Facultad de Derecho de la Universidad Nacional Mayor de San Marcos.

* Abogada por la Universidad Femenina del Sagrado Corazón. Candidata al grado de Magíster en Docencia Universitaria y Gestión Educativa por la Universidad Tecnológica del Perú. Estudios de Derecho Internacional, Religioso Comparado y Humanos en la Universidad de Milán, Italia, Universidad de Montréal, Canadá y Pontificia Universidad Católica del Perú (PUCP). Especialización en Derecho Dominio de 8 idiomas incluido el quechua y manejo de la lengua de señas peruanas. Profesor visitante en la Universidad Autónoma de Chiapas, México. 
negativamente a los magistrados, porque son ellos los que asumen la responsabilidad social de atender casos de violencia doméstica.

Palabras clave: Derecho procesal de familia; Constitucionalización del derecho de familia; Debido proceso y Tutela judicial efectiva; Violencia social y familiar; Prevención de daños y reparación; Evaluación de las condiciones de la víctima; Conflicto familiar

\section{Abstract:}

The context of social violence affects the family sphere, generating a disproportionate increase in statistics in recent years, rendering the actions of the State sterile through its public entities. Public policies to control acts of violence within families are being discredited and the real reason for this failure details a fundamental defect: the State is assuming that violence is fought through legal, judicial and state actions, thereby avoiding addressing the true origin of extreme violence in the country.

As a result of this situation, legislation is observed that is constantly modified, establishing more coercive and punitive levels but without addressing the facts that cause situations of violence, aggressiveness and lack of control of people in their family relationships, because in addition These have been transformed in recent years, generating a symbolic and dysfunctional regulatory system that negatively conditions the magistrates, because they are the ones who assume the social responsibility of dealing with cases of domestic violence.

Keywords : Family procedural law; Constitutionalization of family law; Due process and effective judicial protection; Social and family violence; Damage prevention and repair; Assessment of the victim's conditions; Family conflict

\section{INTRODUCCIÓN}

En los últimos años, el incremento de casos de violencia en el interior de las familias debería generar la necesaria evaluación del panorama criminológico-penal-civil-constitucional-sociológico y familiar peruano (Bermúdez-Tapia, 2019 a, p. 11), pero esta parece ser una premisa ajena a la urgencia de atender un problema nacional, superando las circunstancias coyunturales que usualmente motivan las políticas públicas.

La confusión de acciones en el Estado está provocando el incremento del número de casos de violencia en los hogares peruanos, porque no logra identificar correctamente el marco de acción y por ello se ha limitado a modificar el ámbito normativo penal-familiar provocando una legislación simbólica, 
disfuncional y atemporal que limita la labor de los magistrados porque estos serán los responsables de representar al Estado y no podrán solucionar estas situaciones porque se verán limitados por la legislación que deberán aplicar.

Ante esta situación, el siguiente texto es redactado bajo una metodología hermenéutica de tipo cualitativo con un enfoque sistemático, interdisciplinario y transversal respecto de las acciones del Estado, del Sistema Judicial y de la sociedad peruana, para poder evaluar el margen de acción de las "medidas de protección" establecidas en la Ley № 30364 y su reglamento, sobre la cual se puede identificar el objetivo del texto que consiste en exponer la naturaleza simbólica de la legislación contra la violencia, en particular el que se desarrolla contra la mujer.

\section{UNA LEGISLACIÓN AMPLIA, GENERAL Y PUNITIVA SIN RESULTADOS EFECTIVOS}

Probablemente, el defecto más significativo del Estado sea el considerar que la ley soluciona un problema social y esta premisa parte de la evaluación normativa que se aplica al contexto de la violencia familiar:

Ley 30364 (2015) Ley para prevenir, sancionar y erradicar la violencia contra las mujeres y los integrantes del grupo familiar. En donde se detallan dos elementos referenciales: "erradicar" y la mención a los "integrantes del grupo familiar" pero que excluye la posibilidad de que la violencia se pueda hacer entre dos mujeres, conforme se detalla en el artículo $1^{\circ}$, al detallarse que el objeto de la ley es prevenir, erradicar y sancionar toda forma de violencia producida en el ámbito público o privado contra las mujeres por su condición de tales ..., como si la violencia de madres contra las hijas o la violencia entre hermanas o la violencia entre nietas sobre las abuelas por el acceso a una sucesión son elementos ficticios. Tal como denotan la siguiente normativa:

- Decreto Legislativo № 1186 (2015) que autoriza el uso de la fuerza por parte de la Policía Nacional del Perú.

- Decreto Supremo № 009-2016-MIMP, que es el Reglamento de la Ley № 30364 (2016).

- Decreto Legislativo № 1297 (2017) para la protección de niñas, niños y adolescentes sin cuidados parentales o en riesgo de perderlos.

- Decreto Supremo № 1323-2017 (2017) que fortalece la lucha contra el feminicidio, la violencia familiar y la violencia de género. En donde resulta conveniente detallar que el "fortalecimiento" de las acciones del Estado se ha visto reflejado en el incremento del número de mujeres asesinadas por sus parejas. 
- Ley № 30819 (2018) que modificó el Código Penal y el Código de los niños y adolescentes respecto del feminicidio, al anular la patria potestad de los feminicidas.

- Decreto Legislativo № 1386, que modificó la Ley 30364, ampliando el margen punitivo de la norma.

- Ley № 30862 (2018) que fortalece diversas normas para prevenir, sancionar y erradicar la violencia contra las mujeres y los integrantes del grupo familiar. Donde se observa el énfasis del legislador al insistir en el uso del término "erradicar" pese a que las estadísticas demuestran que sus objetivos son idílicos.

- Decreto Legislativo № 1470 (2020) que se estableció durante la emergencia sanitaria y que a la fecha ha registrado

Conforme esta estructura normativa, parecería ser que el contexto de la violencia doméstica está bajo control estatal porque la "legislación" resulta amplia, específica y literalmente establece condiciones punitivas extremas. Sin embargo, si se analizan las estadísticas del propio Ministerio de la Mujer a octubre del 2020 (MIMP, 2020), es posible detallar:

El número de casos de feminicidios [85 muertes] se ha reducido con respecto del año 2019 [114 muertes], pero especialmente por el efecto de la cuarentena resultante del Decreto Supremo № 044-2020-PCM que declaró el Estado de Emergencia del 16 de marzo al 30 de junio del 2020.

El número de casos de violencia física y psicológica provocadas por la pareja es sumamente significativo, porque en el 2019 reportó el 65\% de casos y en el 2020 reportó el 55\% de casos.

Las regiones con mayores índices de violencia familiar son ciudades con un ámbito urbano superior al rural: Piura, La Libertad, Lima, Callao, Arequipa, Huánuco, Junín y Ayacucho, no ubicándose víctimas fatales en San Martín y en Cerro de Pasco.

Las víctimas por lo general se ubican en la ratio de las edades de 18 a 29 años (42\%) y de 30 a 59 años (44\%) hecho que permite detallar que al exponerse en la legislación el término "integrantes del grupo familiar": No se analizan casos de violencia psicológica contra niños. Las estadísticas del propio Ministerio de la Mujer resultan gráficas en este sentido, porque no se reportan víctimas entre "6-11 años".

Con lo cual presumimos que el Ministerio de la Mujer no ha registrado situaciones de obstrucción de vínculo, alienación parental o casos de 
padectomía que son esencialmente ejecutados por las madres que limitan los derechos del padre cuando este no domicilia junto a sus hijos.

No se analizan los casos de violencia contra personas de la tercera edad, con lo cual no se analizan los casos de "interdicciones" forzadas o de abandono material de estas personas por parte de sus hijos.

No se analizan los casos de violencia familiar contra "hombres", de ninguna edad, siendo estos "personas no sujetas a violencia familiar" conforme las estadísticas mensuales del propio Ministerio de la Mujer.

Sobre estos hechos, que son publicados por el propio Ministerio de la Mujer observamos la ceguera institucional y del propio Estado en evaluar un panorama que se resiste a ser evaluado diligentemente.

\section{LA EVALUACIÓN DE DERECHOS DE GRUPOS VULNERABLES}

Cuando se evalúa el concepto de "víctima" en casos de violencia familiar, se debería dejar de emplear la unidireccionalidad de la legislación porque sólo "ubica" a la mujer como víctima de violencia, excluyéndose de forma malintencionada la violencia que provoca la mujer contra sus propios hijos y contra la que pudiera desarrollar contra su pareja o sus propios progenitores.

Un defecto que se debe en particular a la elevada cifra de violencia física y psicológica contra la mujer, porque esta es una realidad objetiva en el ámbito de la evaluación de la realidad social pero que ha sido interpretada en forma excluyente por el legislador y por eso la legislación no toma en cuenta el contexto de las relaciones en el ámbito familiar en el país.

Por tanto, en este ámbito las principales deficiencias de la legislación son las siguientes:

No se toma en cuenta el contexto de la "crisis familiar" porque se desconoce el ámbito preliminar de la relación familiar disfuncional antes de la ejecución de alguna denuncia en el ámbito penal o de una demanda en el ámbito civil, sobre la materia.

En términos sencillos, el Estado al no "conocer" alguna situación disfuncional en las relaciones familiares no puede intervenir y consecuentemente se genera una cifra negra sumamente elevada que se reporta recién en los fundamentos de hecho de cada denuncia o demanda interpuesta en algún órgano jurisdiccional. 
Las personas que integran relaciones familiares disfuncionales no son conscientes de los alcances de sus derechos, responsabilidades y condiciones para acceder a algún mecanismo de tutela por parte del Estado (López et al, 2004).

Los casos de procrastinación son elocuentes en este sentido y permiten detallar el hecho significativo de que las personas no perciben una condición negativa en su ámbito íntimo o privado.

Una explicación que permite detallar la "justificación" que usualmente exponen las víctimas de violencia familiar que ponderan otras condiciones, como la "familia", "los hijos" o la "propia estabilidad económica" en caso se ejecute una denuncia o una demanda en algún órgano jurisdiccional.

No se toma en cuenta el contexto de la realidad familiar peruana, en particular para superar la premisa excluyente de la "tutela de la familia nuclear y matrimonial".

En este sentido, las personas o parejas que han procreado un hijo (convivientes o concubinatos) no son evaluados correctamente y no se toma en cuenta el proceso de desarrollo de las relaciones familiares en el país, al no tomarse en cuenta los efectos de las familias sucesivas, familias paralelas o familias secuenciales que son los casos más representativos en el ámbito sucesorio en los juzgados nacionales.

No se toma en cuenta el contexto de la dignidad ni de la evaluación de sus alcances, porque el contexto procesal y procedimental pondera más la participación de la "mujer" frente al que pudieran desarrollar los "niños y /o adolescentes" y las "personas de tercera edad".

El varón en este ámbito está excluido de toda valoración y por eso es que inclusive el chantaje económico no es valorado diligentemente en el ámbito de los procesos de alimentos.

No se toma en cuenta la dinámica de una relación de pareja disfuncional. Una referencia que excluye por ejemplo las situaciones de violencia entre personas que son "ascendiente-descendiente" que normalmente se aprecia en el ámbito de la violencia familiar contra personas de tercera edad y las referencias de violencia entre personas ubicadas en el mismo nivel de vínculo familiar, como por ejemplo el que desarrollan "hermanos" con respecto de la sucesión o la tenencia de un bien inmueble de un ascendiente. 
Los alcances de la Casación № 3006-2015, Junín que desarrolló el VIII Pleno Casatorio Civil de la Corte Suprema de la República, detallan este defecto, en particular porque no se logró detallar que las partes procesales eran "medio hermanos".

No se toma en cuenta el contexto subjetivo de las relaciones en una pareja. Referencias como "la reconciliación", o el acuerdo para "retomar la relación" constituyen una referencia muy usual en el ámbito del trámite de alguna denuncia o proceso civil-familiar en el ámbito judicial.

En estas condiciones, ¿la legislación tomó en cuenta esta referencia? Nótese que, en el ámbito extenso de la legislación, el Código Civil sólo detalla estos puntos sólo al momento previo de la expedición de una sentencia en un caso de divorcio y por tanto, para todas las demás situaciones lo que realmente se ejecuta es una analogía muy forzada y extendida, pero porque es promovida por las mismas personas que inicialmente participaron en un hecho de violencia familiar.

En este sentido, a la legislación le faltó "analizar" la realidad socio familiar peruana porque se ha centrado en la literalidad del artículo $4^{\underline{o}}$ de la Constitución, siguiendo el patrón de referencia del Código Civil de 1936 que es seguido por el Código Civil de 1984.

\section{EL CONTEXTO PENAL: SIMBOLISMO, REDUCCIONISMO, TODO Y A LA MISMA VEZ NADA}

La "ley", elemento esencial en la evaluación del presente texto, es un elemento disfuncional porque sus características básicas: general, abstracta, temporal y vinculante no logra ser ejecutiva en el ámbito civil-familiarprocesal y penal en el ámbito nacional (Armenta, 2012).

Al respecto podemos detallar algunos defectos de la legislación penal, que no evalúa el contexto social (Zupi et al, 2011), en este sentido:

\section{A) TIENE ELEMENTOS DE REDUCCIONISMO PENAL.}

La evaluación de los alcances del Decreto de Urgencia № 008-2020 y del Decreto Legislativo № 1459 sobre "alimentos" permite detallar que el segundo proceso seguido por un alimentista y su representante procesal para hacer ejecutiva la prestación de alimentos resulta ineficiente. 
Además de que el mismo "tipo penal" ya queda desnaturalizado por cuanto la "pena" no se ejecuta al plantearse un recurso que pudo haberse ejecutado en el primer proceso judicial de cobro de alimentos, esto es en el juzgado de paz o en el juzgado especializado civil o de familia.

Por tanto, dos procesos penales continuos y vinculados entre sí, resultan extender el "plazo" en el cual se evalúa un derecho natural, humano, fundamental y constitucional en el alimentista, y el legislador no se ha dado cuenta de su error.

En el ámbito de la violencia familiar, la legislación toma elementos del derecho penal del enemigo.

La referencia del artículo 1ำ de la Ley № 30364 “contra las mujeres por su condición de tales" es la representación perfecta de esta línea de evaluación penal, en donde la configuración de un "sujeto activo" de algún delito no podría ser mujer.

\section{B) POPULISMO PUNITIVO.}

Como resultado de los dos puntos preliminares, por cuanto el legislador asume que la promulgación de una norma podrá solucionar situaciones de naturaleza humana, social y familiar disfuncional y en violencia.

\section{C) LAS MEDIDAS DE PROTECCIÓN}

Centrándonos más en la parte más crítica de la Ley № 30364, desarrollaremos el alcance de este recurso procesal en el ámbito familiar, especialmente porque se desarrolla en función a los siguientes puntos:

Permite el desarrollo de principios de orden constitucional, por cuanto está vinculado a la ejecución de los principios de:

Convencionalidad y de la aplicabilidad de Tratados Internacionales, en especial los que protegen a la mujer, a los niños y adolescentes y a las personas de tercera edad.

Constitucionalidad, por cuanto hay una premisa de "tutelar a la familia", sin tener en cuenta que existen varios tipos de familias (BermúdezTapia, 2011 b, p. 44). 
Principio de jerarquía normativa, por cuanto las acciones afirmativas son justificadas en función a la necesidad de limitar los efectos negativos de la violencia contra la mujer, especialmente en el ámbito familiar. Permite identificar y diferenciar el contexto procesal civil del conflicto familiar judicializado.

Esto en función porque en un expediente judicial pueden participar partes procesales pero que no necesariamente son las únicas personas que forman parte de un conflicto familiar judicializado.

En este sentido, la exclusión de las personas que también son afectadas por la violencia desarrollada en el interior de una familia en el trámite de un proceso judicial responde principalmente al alcance de la aplicación del Código Procesal Civil (Bermúdez-Tapia, 2011 a, p. 39).

Consecuentemente, los alcances de la legitimidad para obrar y del interés para obrar deberían ser modificados porque en el contexto familiar en crisis, estos elementos procesales excluyen a determinadas personas que no pueden participar en el proceso judicial de modo activo porque no se les reconocen tal condición.

De modificarse esta condición, con la autonomía del derecho procesal de familia (Bermúdez-Tapia, 2019 c) sería posible superar la exigencia del impulso procesal que suele ser representativo en el ámbito judicial en la especialidad civil-familiar.

\section{D) PERMITE EL DESARROLLO DE GARANTÍAS Y PRINCIPIOS APLICABLES AL ÁMBITO JUDICIAL}

En este sentido se pondera la accesibilidad al sistema de justicia, tratando de reducir los efectos negativos de las condiciones económicas de las partes que sean víctimas de violencia familiar, para tramitar en un plazo razonable un caso de violencia, generándose una acción que procure socializar el contexto familiar en crisis.

Permite el desarrollo de la Tutela Judicial Efectiva y del Debido proceso al desarrollarse los principios de imparcialidad judicial, el registro de casos que permitan el desarrollo de una doble instancia, y que cada decisión judicial sea debidamente motivada, conforme detalla el inciso $5^{\circ}$ del artículo $139^{\circ}$ de la Constitución. 
Sin embargo, el alcance material de la Ley № 30364 no toma en cuenta:

La posibilidad de que existan otras víctimas que no sean las mujeres, niños o niñas o personas de la tercera edad.

En este caso, personas que integran el colectivo LGTBQ o varones no son parte "visible" de este ámbito normativo.

No se toma en cuenta el contexto psicológico y psiquiátrico de las personas que forman parte de estos contextos de violencia y por ello es que resulta imperceptible los casos de:

\section{E) ALIENACIÓN PARENTAL. PADRECTOMÍA.}

Los casos derivados de un comportamiento Doppelgänger (BermúdezTapia, 2019 b, p. 214).

Las situaciones que registran o un síndrome de Estocolmo o de Hibristofilia.

Las situaciones de fertilidad socio múltiple, entre otras situaciones que sólo pueden identificarse a través de procesos de evaluación prolongados y específicos (Bermúdez-Tapia, 2012).

En otro ámbito, la evaluación procesal de las medidas de protección, permiten diferenciarlas de:

Las medidas cautelares, por cuanto en estos casos no se procura garantizar la efectividad de una sentencia, en particular porque el contexto de evaluación puede representar en un futuro próximo o el que las parejas se reconcilien o porque las partes opten por finalizar su relación afectiva (matrimonial-convivencial-concubinal o sexual) sin generar mayores situaciones de violencia.

Por tanto, en este ámbito no se registran riesgos derivados de la demora en la emisión de una sentencia.

Las medidas innovativas o de no innovar, porque en esencia el contexto subjetivo no puede ser detallado por el juez y las partes involucradas en una situación de violencia pueden superar los ámbitos detallados por la legislación. 
Las medidas anticipadas, por cuanto en estos ámbitos resulta materialmente complicado ejecutar una acción inmediata en casos de violencia familiar, tomando en cuenta que una sola declaración puede alcanzar un efecto negativo en la parte denunciada sin que los hechos puedan garantizar la declaración ejecutada inicialmente.

Las medidas autosatisfacías, porque si estas se ejecutan la violencia familiar en esencia habría finalizado y por tanto no podrían ser atendidas en el ámbito judicial.

Consecuentemente, las medidas de protección son un mecanismo procesal, no una institución procesal, que procura neutralizar o limitar los efectos de actos de violencia que se producen en el ámbito familiar, sin importar las condiciones o referencias personales de las personas que ejecutan estos actos, para así garantizar a la víctima un nivel de tutela idóneo a la protección de su dignidad, vida y derechos.

En casos de violencia familiar al acreditarse un daño ¿Es posible reparar dicho daño?

En este punto resulta necesario e imprescindible entender la diferencia entre un conflicto civil puro y un conflicto familiar que se ha judicializado porque los contextos son autónomos y esto es porque la naturaleza del conflicto es especial en cada caso.

Sin embargo, en este ámbito, la reparación a los daños resulta una figura mal interpretada en el ámbito procesal civil porque surgen una serie de variables que merecen un análisis individual.

Respecto de la identificación del "daño".

En el ámbito de las relaciones familiares, el impacto negativo de una situación de violencia permite detallar que podrían surgir varias referencias y todas ellas podrían quedar anuladas por las mismas partes en conflicto porque no siempre se podrá ejecutar una evaluación objetiva sobre el "daño".

Así una separación o un divorcio provocada por una situación de violencia doméstica tendrá una percepción subjetiva diferente de una separación o divorcio provocada por un adulterio, respecto de la víctima y ante ello surge la cuestión ¿Cómo reparar ambos tipos de daño? 
El proyecto de vida matrimonial resulta disfuncional en un contexto contemporáneo porque esta misma evaluación no se produce en forma similar cuando se analizan casos, como:

El engaño, adulterio o infidelidad de la mujer respecto de la procreación de un hijo que no sea de la pareja y donde se haya ejecutado su reconocimiento que luego se impugna con la acreditación de la verdad filiatoria que detalla una prueba biológica.

En estos ámbitos, la doctrina nacional sólo ha desarrollado el primer punto de estos dos casos y ha negado la opción del segundo caso, como si el alcance del artículo $145^{\circ}$ del Código Penal no existiera.

Respecto de la temporalidad del daño. La regla general en las parejas que optan por no continuar su relación afectiva sin identificar si se trata de matrimonio, convivencia, concubinato o relación afectiva-sexual no siempre es evaluada diligentemente por ambas partes y por ello es que la ejecución de un divorcio es la excepción, cuando en esencia debería ser la regla.

Inclusive con la reforma del Código Civil, con el Decreto Legislativo № 1377, donde se eliminó la presunción de paternidad por parte del cónyuge resulta incongruente con la realidad familiar, por cuanto la mujer casada no está obligada a indicar quien es el progenitor de su hijo, como si tal referencia no fuera importante.

Los derechos del niño procreado parecen se relativizan en estos ámbitos de forma incongruente con el alcance de la Ley № 30364 porque en estos casos, la persona que atenta contra un derecho a la identidad, a la dignidad, a la pertenencia a una familia, es la propia mujer.

Finalmente surge una condición final, ¿Cómo reparar un daño? Si regresar a una etapa previa a los hechos resulta ser una condición imposible de ejecutar.

\section{CONCLUSIONES}

De lo detallado, surge la necesaria evaluación crítica de los alcances de la legislación sobre violencia familiar, porque el nivel de simbolismo punitivo resulta ser el principal defecto que amplifica las situaciones de violencia familiar en el país, provocando una condición que sólo la asumen los magistrados en el país, sin tener en cuenta que el contexto normativo es el que resulta disfuncional. 
En este sentido, las medidas de protección son un instrumento procedimental que no garantiza el cese de la violencia porque el contexto de la crisis familiar no ha sido debidamente evaluado y esto porque la ley asume que el contexto de las personas no resulta importante y permite que el juez no ejecute una inmediación judicial acorde a las circunstancias y se asuma que una medida judicial solucionará el conflicto sobre la cual la ley ha procurado una acción de "erradicación".

Finalmente, recomendamos que exista un programa de evaluación, seguimiento y ayuda a las familias, ya que las medidas de protección son insuficientes para garantizar su estabilidad y seguridad. Por el contrario, este es un problema social no legislativo, ya que los conflictos familiares nacen de disputas y controversias interpersonales, los mismos que se pueden resolver de manera pacífica, siempre y cuando se les enseña a las familias que esta es la solución antes que la violencia o cualquier tipo de acto que denigre su dignidad como individuos.

\section{REFERENCIAS}

Armenta Deu, T. (2012). Sistemas procesales penales: la justicia penal en Europa y América ¿Un camino de ida y vuelta? Madrid: Marcial Pons

Bermúdez-Tapia, M. (2011 a). La constitucionalización del derecho de familia. Lima: Ediciones Caballero Bustamante

Bermúdez-Tapia, M. (2011 b). Redefiniendo el derecho de familia en la tutela del vínculo familiar en la jurisprudencia peruana. Revista de Derechos Fundamentales. Universidad Viña del Mar. (5), 43-62

Bermúdez-Tapia, M. (2012). Derecho procesal de familia. Aproximación crítica no convencional a los procesos de familia. Lima: Editorial San Marcos

Bermúdez-Tapia, M. (2019 a). La evaluación constitucional de derechos en el derecho de familia. Lima: Gaceta Jurídica

Bermúdez-Tapia, M. (2019 b).. Elementos procesales y probatorios en el derecho de familia. Bogotá: Ediciones Nueva Jurídica.

Bermúdez-Tapia, M. (2019 c) La autonomía del derecho procesal de familia en función a la atención de los conflictos familiares judicializados, en Torres Carrasco, M. (Director) Derecho procesal de familia. Lima: Gaceta Jurídica, 389-436 
López, R. y Pérez, A. (2004.) Tendencias actuales en el derecho de familia. Almería: Universidad de Almería

Ministerio de la Mujer y Poblaciones Vulnerables (2020, 07 de octubre). Programa Nacional para la prevención y erradicación de la violencia contra las mujeres e integrantes del grupo familiar. https:/ / www.mimp.gob. pe/contigo/contenidos/pncontigo-articulos.php? codigo=39

Zupi, M. y Estruch, E. (2011). Desafíos de la cohesión social en tiempos de crisis: Diálogo Euro-Latinoamericano. Madrid: Universidad Complutense de Madrid

Fecha de recepción: 12-09-2020

Fecha de aceptación: 19-10-2020 